\title{
Nevirapine Induced Exfoliative Dermatitis: A Case Report
}

\author{
Dr.Ramya. Rachamanti M.B.B.S (M.D.) ${ }^{1}$ Dr.Mattam Bheemesh Naidu M.D. ${ }^{2}$ \\ Dr.Keelu Raj kumar M.B.B.S (M.D.) ${ }^{3}$ \\ ${ }^{1,2,3}$ (Department of Pharmacology, Guntur Medical Collage.Dr. NTR University of Health Sciences, India)
}

\begin{abstract}
:
Introduction:- To report a case of Exfoliative Dermatitis in an individual with Acquired Immuno Deficiency Syndrome who is taking Nevirapine.

Method: -A 25-year-old female came to Anti Retroviral Therapy Unit of Government General Hospital, Guntur with a complaint of fever, itching and scaling of skin involving both the palms. Drug history revealed that she was prescribed with Highly Active Anti Retroviral Therapy which includes Zidovudine,Lamivudine and Nevirapine as a combination regimen she was sent to dermatology outpatient unit for consultancy.

Dermatologist diagnosed this condition as Exfoliative Dermatitis clinically and sent the skin scraping over the palm for histopathological examination. The condition improved with stoppage of Nevirapine. So, we attributed this Adverse Drug Reaction to Nevirapine.
\end{abstract}

Result:-Histopathological examination confirmed that the skin rash as Exfoliative Dermatitis

Conclusion: - A strict vigilance of Adverse Drug Reaction is required in anti retroviral therapy unit of every hospital

Keywords: AIDS-Acquired Immuno Deficiency Syndrome, ART-Anti Retroviral Therapy Unit, ED - Exfoliative Dermatitis, HAART - Highly active antiretroviral therapy, Nevirapine.

\section{Introduction:-}

Exfoliative dermatitis ${ }^{1}$ (ED) is an inflammatory skin disease with scaling that affects nearly the entire cutaneous surface. The common causes ${ }^{2}$ of erythroderma is exacerbation of an underlying skin disease or a drug reaction .The Incidence of drug reaction is $28 \%$

The patient in this case has no history of any skin disease. So, there is more chance that it is induced by drug. Drugs ${ }^{3}$ which cause ED are Allopurinol, Nevirapine ${ }^{4}$ Carbamazepine, Codeine,Captopril, Diphenylhydantoin Gold, Antimicrobials etc. Among these drugs, the drug history revealed that the patient is taking only Nevirapine .So, Nevirapine is the cause for Exfoliative dermatitis in this case.

Nevirapine, a dipyridodiazepinone, non-nucleoside reverse transcriptase inhibitor ${ }^{5,6}$ is widely prescribed as a part of the combination therapy of Human Immunodeficiency Virus because of its efficacy and good tolerability.

The common adverse drug reactions observed with nevirapine includes skin rashes ${ }^{7}$ and Hepatotoxicity ${ }^{8}$. The skin rashes are usually mild and occur in initial 6-12 weeks of starting the therapy. Incidence of severe form of skin rash is $0.5-1 \%^{4}$ The severe form of skin rash ED has been reported once in Government General Hospital. Here, I am reporting that case of Exfoliatiave Dermatitis secondary to Nevirapine.

\section{Case Report:-}

A 25 year old female patient came with a complaint of fever, itching, scaling in both her palms. She was a known Acquired Deficiency Syndrome patient. She was diagnosed to have Acquired Deficiency Syndrome 2 yrs before. She was prescribed with Highly Active Anti Retroviral Therapy with zidovudine 300 mg plus lamivudine $150 \mathrm{mg}$ plus nevirapine $200 \mathrm{mg}$ since then. Nevirapine was advised once daily for first two weeks and twice daily thereafter. After ten weeks of treatment, the patient developed erythematous pruritic patches with fine white scaling over the patches that progressed over next two weeks to attain the present diffuse pattern of exfoliation involving both the palms. On detailed examination, diffuse exfoliative erythematous intensely pruritic patches were present involving more than $70 \%$ of the body surface area, more severe in the palms and soles (Fig 1). The conjunctivae, skin over the scalp and genitalia were spared. The body temperature was $39.5^{\circ} \mathrm{C}$. Psoriasis, phyto-photo dermatitis, atopic dermatitis, seborrheic dermatitis, and contact dermatitis were excluded on the basis of detailed history and clinical grounds. The condition was diagnosed by consultant dermatologist as drug induced-Exfoliative dermatitis by suspecting nevirapine as the causative agent on Investigations revealed HIV-reactive, Heamoglobin level as 7.8 grams or $54 \%, \mathrm{CD}_{4}$ count is $325 \mathrm{cells} / \mathrm{cc}$, Blood count, random blood sugar, chest X-ray, liver and renal function tests were normal. Histopathological examination revealed ED 
(Fig 2)

The treatment is dependent on the cause. The patient was advised to discontinue nevirapine and she was prescribed alternative therapy with efavirenz and patient was treated for two weeks with T.Predisolone T.paracetamol $650 \mathrm{mg}$ thrice daily, and betamethasone $0.05 \%$ with glycerin lotion applied thrice daily on the affected areas. The patient showed significant improvement at the end of two weeks and was discharged after starting a new highly active anti retroviral treatment (HAART) regimen zidovudine $300 \mathrm{mg}$ plus lamivudine 150 mg plus efavirenz 600md once daily. In subsequent fortnightly follow-up for next 30 days, the patient showed gradual and complete resolution of the lesions with no recurrence of previous symptoms and tolerated the new HAART regimen well

\section{Discussion:-}

The appearance of ED in this patient after Ten weeks of initiation of nevirapine-based initial regimen, resolution of the symptoms following withdrawal and patient tolerating the modified HAART regimen ${ }^{9}$ with efavirenz instead of nevirapine without any adverse reactions for next 30 days clearly suggested that nevirapine a causal drug for ED in this patient, suggesting a temporal relationship to nevirapine administration. This adverse reaction is not dose-related, can be labeled as a type-B(bizarre) ${ }^{10}$ class of adverse effect and can be considered as probable or likely as per WHO scale of causality assessment ${ }^{11}$ of suspected adverse drug reactions.

Nevirapine is indicated for the primary treatment of HIV, administered along with NRTIs and/or protease inhibitors. It has no cross resistance or cross reactivity with NRTIs. Since the drug is a moderate autoinducer of its metabolism, it is initiated with a lead-in dose of $200 \mathrm{mg} /$ day for first 14-days and then escalated to $400 \mathrm{mg} / \mathrm{day}$. This mode of initiation has also been shown to lessen the frequency of rash ${ }^{1} \mathrm{The}$ overall incidence of ED ranges from 0.0009 to $0.071 \%$, wherein drugs are implicated in $4 \%-39 \%$ of the cases. The mortality rate associated with ED is $3.75 \%-64 \% .{ }^{12}$ HIV infected patients are found to be at a higher risk of developing drug allergy ${ }^{13}$. Hence, a strict vigilance ${ }^{14}$ of adverse drug reactions is necessary in HAART especially in the initial months of therapy.

\section{Conclusion:-}

Exfoliative dermatitis is a serious and life threatening disease ${ }^{15,16}$ in which some of cases are drug induced and are preventable.

The adverse drug reactions need to identified as early as possible and rational alternate therapy should be given to avoid the complications and to improve patient compliance.

Recently it was found that HLA B*3505 allele ${ }^{17}$ as a strong predictor for nevirapine induced skin reactions in HIV infected patients. So,cost effective methods should be developed to diagnose HLA B*3505 allele to prevent drug reactions.

\section{Figure legends:-}

Figure 1: Intense exfoliation and fine white scaling on the patient's palms

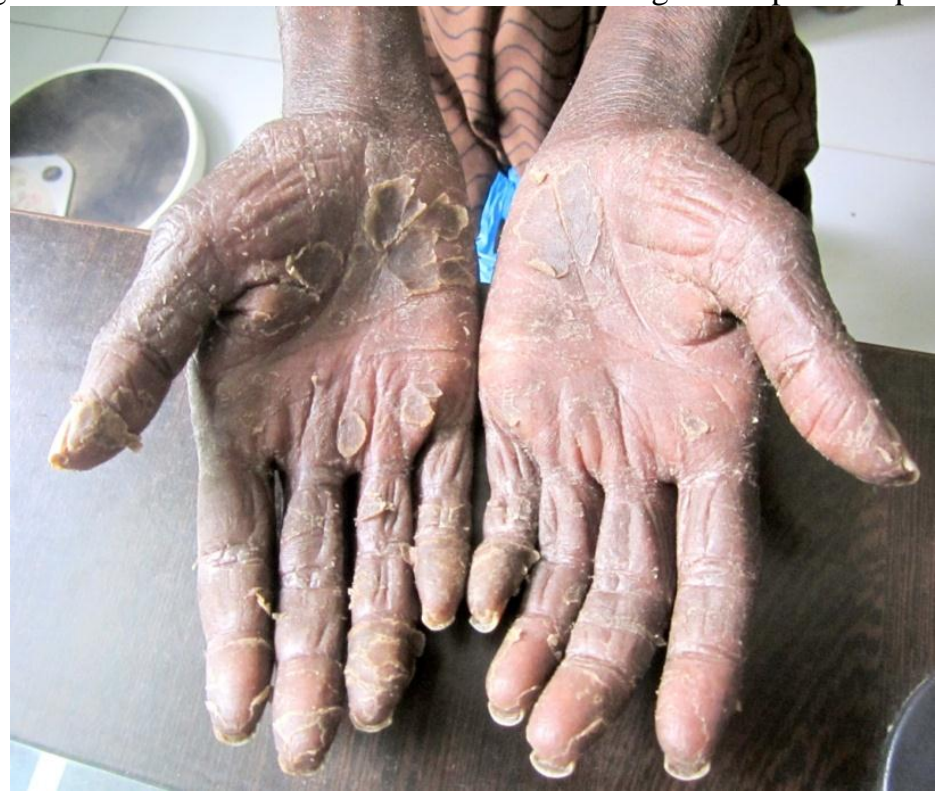


Figure 2 : Histopathological examination of ED

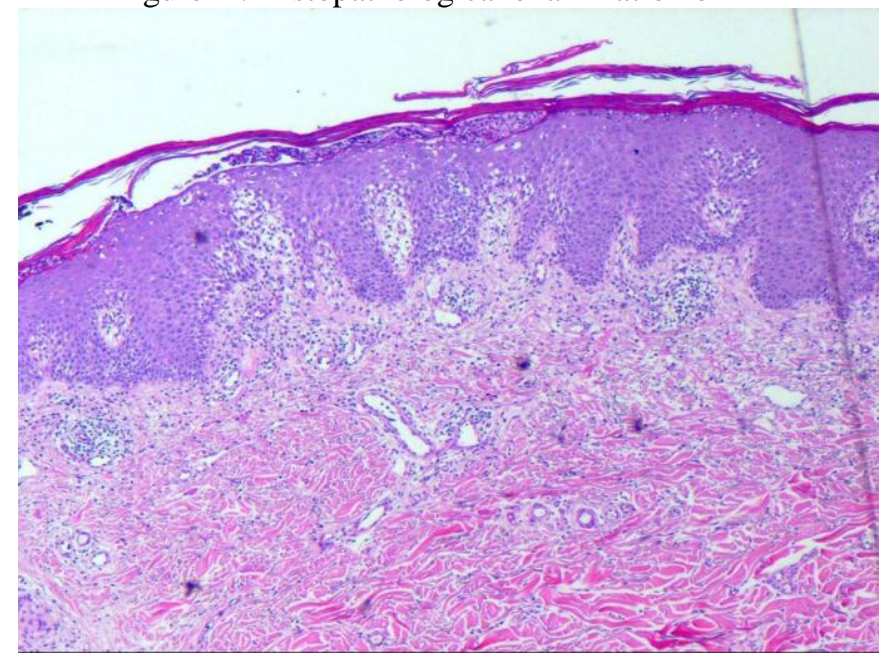

Acknowledgement:-

My heartfelt gratitude towards Dr.Zaheda Bano, Head of the department of Pharmacology and Dr. Jyothi, medical officer of ART unit of Govt General Hospital,Guntur for the valuable guidance and immense support they lent me in bringing out this work.

\section{References}

[1] Freedberg, et al. (2003). Fitzpatrick's Dermatology in General Medicine. (6th ed.). McGraw-Hill.

[2] Yuan XY, Guo JY, Dang YP, Qiao L, Liu W. Erythroderma: A clinical-etiological study of 82 cases. Eur Dermatol. May-Jun 2010;20(3):373-7.

[3] V.N.Sehgal Exfoliative dermatitis j.dermatol v 173 no 6 19861986;173:278-284

[4] Aronson JK. Meyler's side-effects of drugs. J.Elsevier; 15th ed. Vol. 4. 2006. pp. 2498-502.

[5] Goodman and Gilman's: The Pharmacological basis of therapeutics. 10th ed. New York: McGraw-Hill; 2006. pp. $1273-314$.

[6] Patel SS, Benfield P (Oct 1996). "New drug profile: nevirapine".J. Clinical Immunotherapeutics 6 (4): 307-317

[7] Stuart Johnson, Adverse effects associated with use of Nevirapine in HIV JAMA. 2000:284(21):2722-2723

[8] Katzung Basic and clinical pharmacology. 11th ed. New York: McGraw-Hill; 2009. pp. 845-75.

[9] Ritter, J M (2008). A Textbook of Clinical Pharmacology and Therapeutics. Great Britain. p. 62. .

[10] Edwards IR, Aronson JK. Adverse drug reactions: Definitions, diagnosis and management. Lancet.2000;356:1255-9.

[11] Side ahmed adverse drug reaction and causality assessment J.Lung India. 2011 Apr-Jun; 28(2): 152-153.

[12] Fitz Patrick's Textbook of dermatology in general medicine.7th ed. Vol. 1. New York: McGraw-Hill; 2008. pp. 225-32.

[13] Harrison's Principles of internal medicine. 17th ed. Vo 1. 1. New York: McGraw-Hill; 2008. pp. 343

[14] Sara Gambrill, , China's Pharmacovigilance System: The Hunger For Safety Insights, Clinical Leader, December 7, 2011

[15] Byer RL, Bachur RG. Clinical deterioration among patients with fever and erythroderma. Pediatrics. Dec 2006;118(6):2450-60

[16] Bruno TF, Grewal P. Erythroderma: a dermatologic emergency. CJEM. May 2009;11 (3):244-6

[17] Chantarangsus, Mushiroda, T, “ HLA-B*3505 is a strong predictor for nevirapine-included skin adverse drug reactions HIVinfected Thai patients “ Pharmacogenet Genomics 2009 FEB : 19(2): 139-146 